\title{
Research on Fuzzy Inventory Control under Supply Chain Management Environment
}

\author{
Guangyu Xiong and Hannu Koivisto \\ Automation and Control Institute, \\ Tampere University of Technology, P.O. Box 692, \\ FIN-33720 Tampere. Finland \\ xgy@ac.tut.fi
}

\begin{abstract}
The inventory control has been paid great attention for a long time because of its important in the cost control. This paper presents an approach to use fuzzy control system applied in controlling inventory under supply chain management. A fuzzy control system based on the (s, S) framework is given, and the fuzzy controller in inventory control makes it easier to design the system. Two inventory control approach are applied in two cases study, which are single stage inventory and multiple stage inventory in supply chain. For each case, the inventory control approaches are used based on the give data. The cases are given to illustrate the design procedure of the fuzzy controller and its effectiveness. Some conclusions are drawn out based on the simulation.
\end{abstract}

\section{Introduction}

The inventory control has been paid great attention for a long time because of its important in the cost control since Ford Harris' famous EOQ model was first given in 1913[1]. One of the objectives is to minimize the total expected inventory costs per unit time while satisfying the customer demand on time. Under the influence of the supply chain management, traditional inventory control theories and methods are no longer adapted to the new environment. So it will have great practical implication to find new methods for inventory control.

In this paper, we analyse and discuss an approach to use fuzzy control applied in inventory control under supply chain management based two cases, which are the single stage inventory and the multiple stage inventory in supply chain, where the demand process is uniform distribution. Firstly, the classic inventory control methodologies is discussed, where the classic inventory model cost equation is given. Meanwhile, a fuzzy control system based on the (s, S) framework is introduced. The core advantages of a fuzzy controller are robustness under uncertainty, expert experiments considered, and inaccurate information. Therefore, the fuzzy controller in inventory control makes it easier to design the system. Next, we apply two inventory control approach to two cases, the fuzzy control system will be compared with the classical inventory control system for each case. Then, both systems were simulated over a 
span of the demand periods for each case using Matlab. The design procedure and fuzzy control effectiveness are illustrated. We compared the fuzzy control system with the classical control system for the different aspect. Finally, some conclusions are drawn out based on the simulation.

\section{The Inventory Control Approaches}

Inventory is considered as an accumulation of materials or product that will be used to satisfy future demand for materials or product.

Inventory requires the policy to mange the inventory, which may involve the quantitative problems dealing with controlling material are [6]:

- The amount of raw material ordered,

- Time to order raw material,

- Right method used,

- A minimum cost.

Obvious the cost involves the related to the previous actions, i.e. three factors contribute to inventory costs:

- Carrying Cost

Cost of storage and space,

Costs incurred from taxes and insurance,

Cost of obsolescence.

- $\quad$ Shortage Cost

Cost of lost sales and good will.

- $\quad$ Ordering Cost

Cost of preparing, placing orders,

Cost of shipping and handling.

\subsection{The Classic Inventory Control Approach}

As mentioned above, the choice of the policy will be done with the objective of the minimum costs. An inventory policy is the review and ordering discipline used to control inventory.

The most common policies are: periodic review, which need to review a new order of the amount specified by the order quantity at equal interval of time [2]. Inventory levels are observed at equal intervals of time T. Policy defined by:

$$
\mathrm{Q}_{\mathrm{i}}=0 \quad \text { if } \mathrm{I}_{\mathrm{i}}>\mathrm{s} \text {. }
$$

Or

$$
\mathrm{Q}_{\mathrm{i}}=\mathrm{R}-\mathrm{I}_{\mathrm{i}} \quad \text { if } \mathrm{I}_{\mathrm{i}} \leq \mathrm{S} \text {. }
$$

Where:

$\mathrm{R}=$ target inventory level when order placed

$\mathrm{S}=$ reorder level 
$\mathrm{Ii}=$ inventory level at end of period $\mathrm{i}$

$\mathrm{Qi}=$ order size at period $\mathrm{i}(\mathrm{R}-\mathrm{Ii})$

Where no order is placed when the inventory level is greater than the reorder level; and an order quantity of (R - Ii) is placed when inventory level is at or below the reorder level.

Three parameters define this policy: R, r, and T. The optimal values for $\mathrm{R}, \mathrm{r}$, and $\mathrm{T}$ are determined to minimize total inventory costs. We can calculate the general inventory quantity for periodic review as the following equation:

$$
\text { Invtoday }(\mathrm{t}+1)=\operatorname{Invtoday}(\mathrm{t})-\operatorname{Demand}(\mathrm{t})+\operatorname{Order}(\mathrm{t}) \text {. }
$$

Where:

Invtoday $(\mathrm{t}+1) \ldots$ Next inventory quantity;

Invtoday $(\mathrm{t}) \_$C Current inventory quantity;

$\operatorname{Order}(\mathrm{t}) \_$Current order quantity.

We can summarize the total cost of a general inventory model for periodic review as a function of its principal component in the following manner [2]:

$($ Total inventory cost $)=($ purchasing cost $)+($ setup cost $)+($ holding cost $)+($ shortage cost)

In practice, however, that an inventory model need not include all four types of costs, either because some of the costs are negligible or will render the total cost function too complex for mathematical analysis, so we can delete a cost component only if its effect on the total cost model is negligible. For this paper, we just calculate the total inventory costs using the following cost equation:

$$
\mathrm{C}_{\mathrm{T}}=\mathrm{C}_{\mathrm{h}}+\mathrm{C}_{\mathrm{o}}+\mathrm{C}_{\mathrm{s}} \text {. }
$$

Where:

$\mathrm{C}_{\mathrm{T}}$ _ Total inventory cost

$\mathrm{C}_{\mathrm{h}}$ __ Total holding cost

$\mathrm{C}_{\mathrm{o}}$ __ Total ordering cost

$\mathrm{C}_{\mathrm{s}} \_$Total shortage cost

\subsection{Fuzzy Model}

As stated previously, a classical ( $\mathrm{s}, \mathrm{S}$ ) policy is: at the moment when the inventory drops below level s, order units up to level $S[1,4]$. This implies there is always a fixed number $\mathrm{Q}=\mathrm{S}$-s units ordered. But for desecrate case, if the inventory level is lower than s, place an order. It's possible that the inventory level drops to zero before the new order arrives. We present an improvement approach inventory control for the supply chain using fuzzy control system. With this approach, several aspects of the system were handled in the same manner as in the crisp runs. The number of orders, number of stockouts, and average inventory were all calculated in the same way. The fuzzification occurred when an order was being placed. 
When the inventory level was dropped below a point (inventory level), the inventory level and the current period's demand were given membership function values. The membership values were based on the logic described below. To maintain flexibility in the model, all the parameters indicated below are in terms of the model's inputs. This allows the model to adapt to different cases. The core advantages of a fuzzy controller are robustness under uncertainty, expert experiments considered, and inaccurate information.

Fuzzification. We classify the demand, inventory level and order into the three sorts: low, medium and high. The corresponding the membership function is shown in fig. 1 .

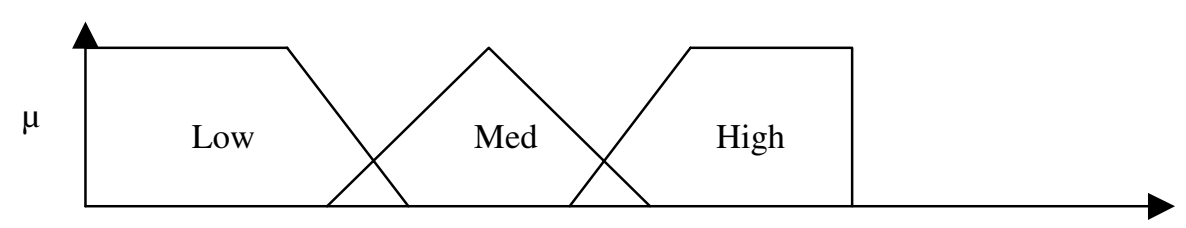

Fig. 1. Membership function

The values on the $\mathrm{x}$-axis represent the different values for different variables. The scalar factor could be changed easily. Varying the value of this scaling unite can be tuning the membership function to make the performance better.

Fuzzy Control Rules. The fuzzy control rules are based on the experiences on inventory ordering policy. The relationship between demand, current inventory level and the amount to be ordered is summarized in Table 1. This table should be read "If demand is High, and the inventory level is Medium, then order High amount of items."

Fuzzy Operators. Selection of the intersection and union operators was constrained by the need to reduce the frequency of ordering. If the operators were compensatory, that could cause the resulting purchase amounts to be further from the extremes, eventually causing an increase in the frequency of orders. This would increase the cost of the system. Another factor of interest was computing cost; complex operators drastically increase the number of computations necessary to run the system. For these reasons, the maximum and minimum operations were selected as the union and intersection operators.

Table 1. Demand, Inventory Level and Order Amount Relations

\begin{tabular}{|c|c|c|c|}
\hline & \multicolumn{3}{|c|}{ Demand } \\
\hline Inventory Level & Low & Medium & High \\
\hline Low & High & Med & High \\
\hline Medium & Med & High & High \\
\hline High & Low & Low & High \\
\hline
\end{tabular}




\section{Case Study}

To compare the performance and implementation of the fuzzy and classical inventory control systems. We apply two inventory control approach to two cases, which are the single stage inventory and the multiple stage inventory in supply chain (Figure 2). Our supply chain looks like the following [6]:

We are a distributor for a certain product. The demand for our product comes from a single source, a retailer (for case 1) or multiple sources, three retailers (for case 2). We get supplied the product from a manufacturer, whom we order from whenever our inventory is at a certain level. For the multiple stage inventory problem, the manufacturer in turn gets supplied from a vendor.

We make the assumptions for the cases:

- The demand has a uniform distribution. Demand is a function of $t$, which is Demand ( $\mathrm{t}$ ).

- Average daily demand (D) from past data.

- The manufacturer (for case 1) or the vendor (for case 2) is a reliable supplier.

- About delay time: For the singer stage inventory, the delay time for us to receive an order after the order had been placed with the manufacturer is some days (delay). For the multiple stage inventory, the delay time to receive an order after the order had been placed is some days (delay).

- Initialize inventory: For the singer stage inventory, to cover the first delay days, initialize inventory by: inv $=\mathrm{D} *$ delay. For the multiple stage inventory, initialize inventory based on D, delay, and number of stages from reliable source.

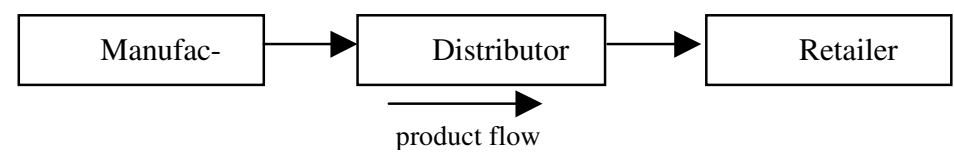

(a) Case 1: Single Stage Inventory

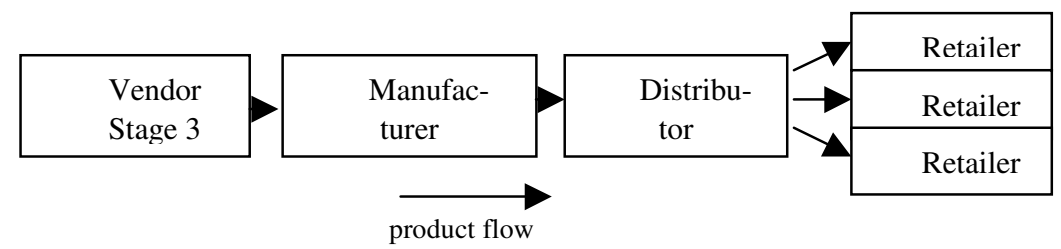

(b) Case 2: Multiple Stage Inventory

Fig. 2. Inventory under supply chain management environment 


\section{Simulation}

Because the order is placed in the beginning of the period, we don't know the exact demand, we create the demand based on its distribution functions. In Matlab, we use rand ( ) to create random values for demand at every period and store the values in an array. The simulation could be based on the cost evaluation equation (4) used the following data [4]:

$$
\mathrm{C}_{\mathrm{T}}=\mathrm{C}_{\mathrm{h}}+\mathrm{C}_{\mathrm{o}}+\mathrm{C}_{\mathrm{s}}=\mathrm{h} \times \mathrm{Q}+\mathrm{K} \times \mathrm{N}+p \times \text { (numbers of Stockouts). }
$$

Where:

$\mathrm{N}=$ number of times of ordering

$\mathrm{Q}=$ average inventory quantity

$\mathrm{K}=$ setup cost for placing an order

$\mathrm{K}=12000 \$$

$\mathrm{h}=$ holding cost per unit inventory per unit time $\mathrm{h}=0.3 \$ /$ unit/period

$\mathrm{p}=$ shortage or stockout cost $\mathrm{p}=5 \$ /$ unit stockout

Table 2. Simulation for singer stage inventory

\begin{tabular}{|c|c|c|c|c|c|c|}
\hline \multirow{2}{*}{ Series } & \multicolumn{2}{|c|}{ Fuzzy Inventory Controller } & \multicolumn{2}{|c|}{ Classical Inventory controller } \\
\cline { 2 - 7 } & $\begin{array}{c}\text { Total } \\
\text { cost }\end{array}$ & $\begin{array}{c}\text { Order } \\
\text { times }\end{array}$ & $\begin{array}{c}\text { Average } \\
\text { inventory }\end{array}$ & Total cost & $\begin{array}{c}\text { Order } \\
\text { times }\end{array}$ & $\begin{array}{c}\text { Average } \\
\text { inventory }\end{array}$ \\
\hline 1 & $\begin{array}{c}8.0640 \mathrm{e} \\
+005\end{array}$ & 67 & $\begin{array}{c}8.0029 \mathrm{e}+ \\
003\end{array}$ & $\begin{array}{c}2.2462 \mathrm{e}+ \\
006\end{array}$ & 187 & $\begin{array}{c}7.4120 \mathrm{e}+ \\
003\end{array}$ \\
\hline 2 & $\begin{array}{c}8.9036 \mathrm{e} \\
+005\end{array}$ & 74 & $\begin{array}{c}7.8758 \mathrm{e}+ \\
003\end{array}$ & $\begin{array}{c}2.4742 \mathrm{e}+ \\
006\end{array}$ & 196 & $\begin{array}{c}7.2187 \mathrm{e}+ \\
003\end{array}$ \\
\hline 3 & $\begin{array}{c}8.5436 \mathrm{e} \\
+005\end{array}$ & 71 & $7.85940 \mathrm{e}+$ & $2.4622 \mathrm{e}+$ & 205 & $7.2713 \mathrm{e}+$ \\
& & 003 & 006 & & 003 \\
\hline
\end{tabular}

Table 3. Simulation for multiple stage inventory

\begin{tabular}{|c|c|c|c|c|c|c|}
\hline \multirow{2}{*}{ Series } & \multicolumn{3}{|c|}{ Fuzzy Inventory Controller } & \multicolumn{2}{|c|}{ Classical Inventory controller } \\
\cline { 2 - 7 } & $\begin{array}{c}\text { Total } \\
\text { cost }\end{array}$ & $\begin{array}{c}\text { Order } \\
\text { times }\end{array}$ & $\begin{array}{c}\text { Average } \\
\text { inventory }\end{array}$ & $\begin{array}{c}\text { Total } \\
\text { cost }\end{array}$ & $\begin{array}{c}\text { Order } \\
\text { times }\end{array}$ & $\begin{array}{c}\text { Average } \\
\text { inventory }\end{array}$ \\
\hline 1 & $\begin{array}{c}9.5160 \mathrm{e} \\
+006\end{array}$ & 793 & 59.6206 & $\begin{array}{c}1.1904 \mathrm{e} \\
+007\end{array}$ & 994 & 42.6793 \\
\hline 2 & $\begin{array}{c}9.5040 \mathrm{e} \\
+006\end{array}$ & 792 & 61.8706 & $\begin{array}{c}1.0560 \mathrm{e} \\
+007\end{array}$ & 880 & 42.4915 \\
\hline 3 & $\begin{array}{c}9.4800 \mathrm{e} \\
+006\end{array}$ & 790 & 65.3649 & $\begin{array}{c}1.1928 \mathrm{e} \\
+007\end{array}$ & 994 & 42.6873 \\
\hline
\end{tabular}




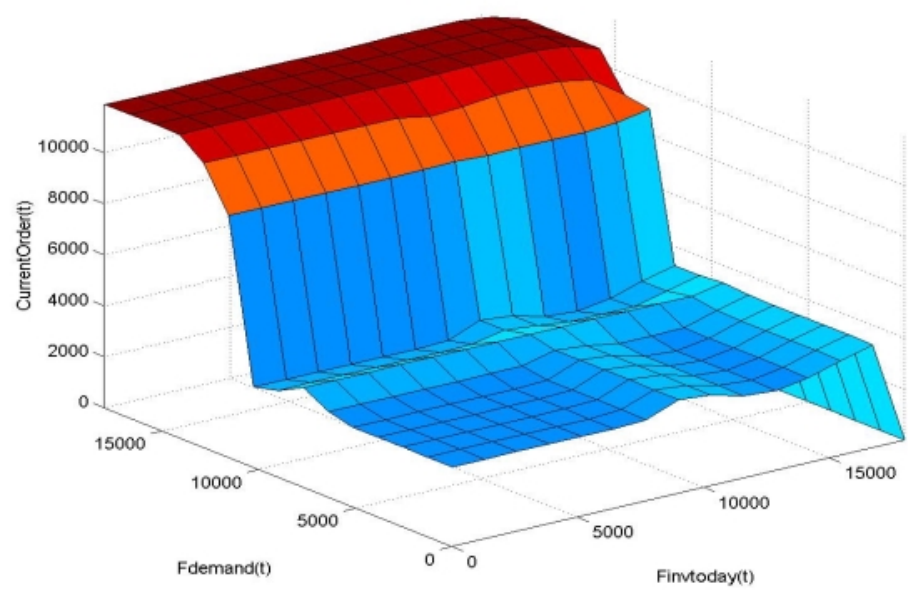

Fig. 3. Control surface for the singer stage inventory
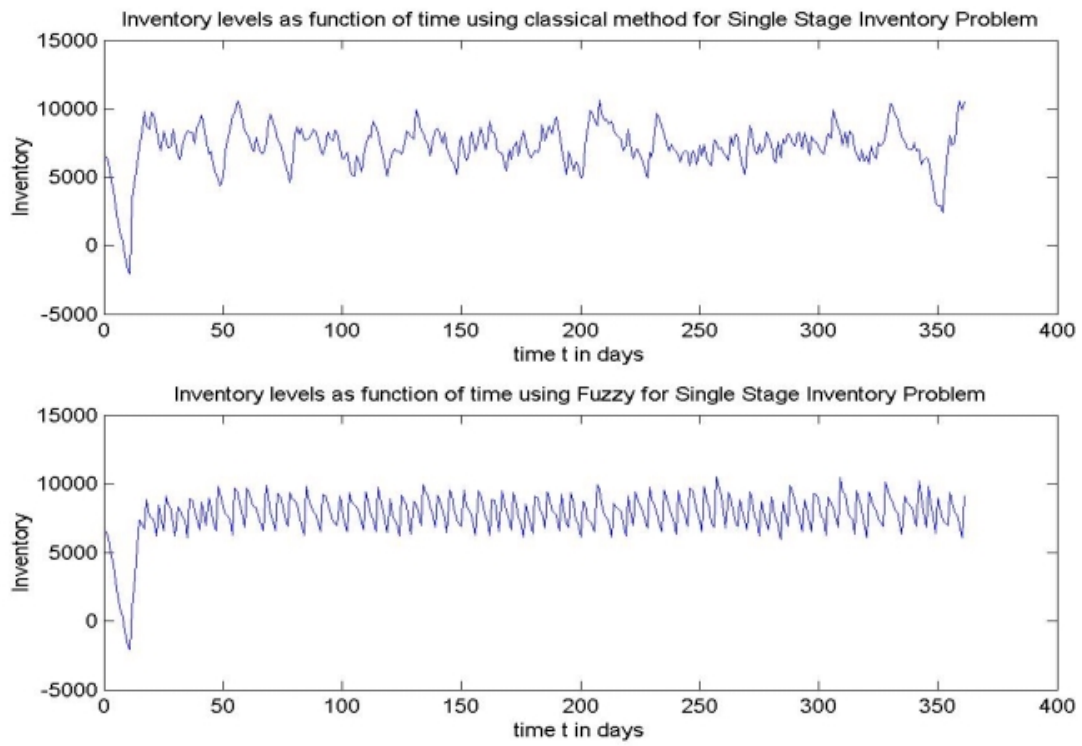

Fig. 4. Case 1: Inventory curve for using fuzzy and classical approach 

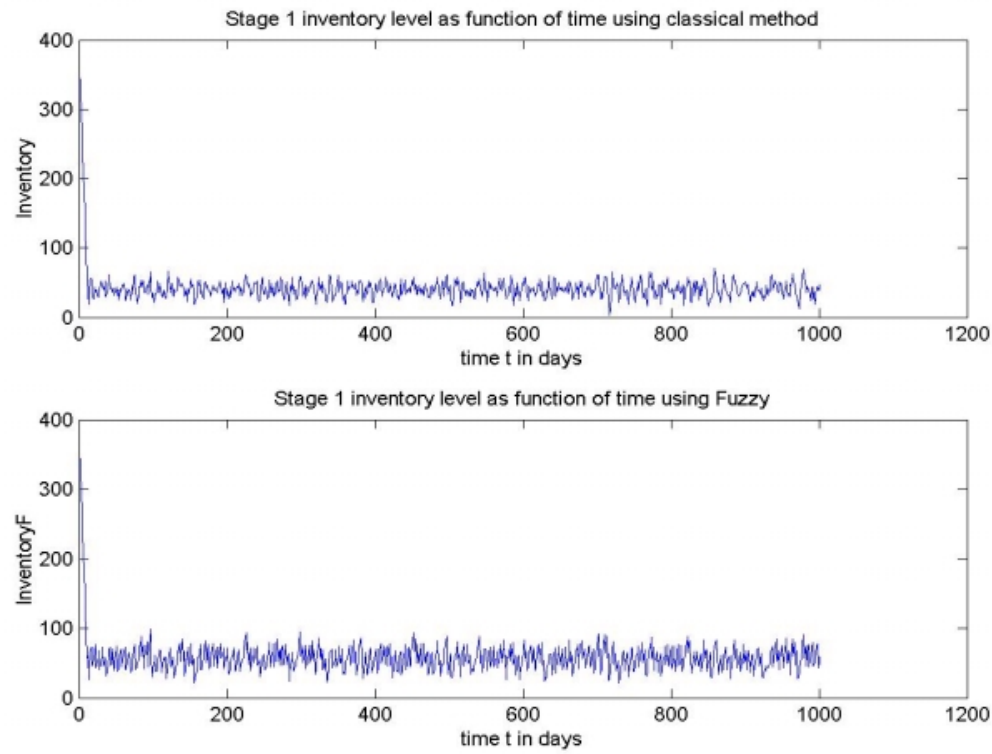

Fig. 5. Case 2: Inventory curve of stage1 using fuzzy and classical approach
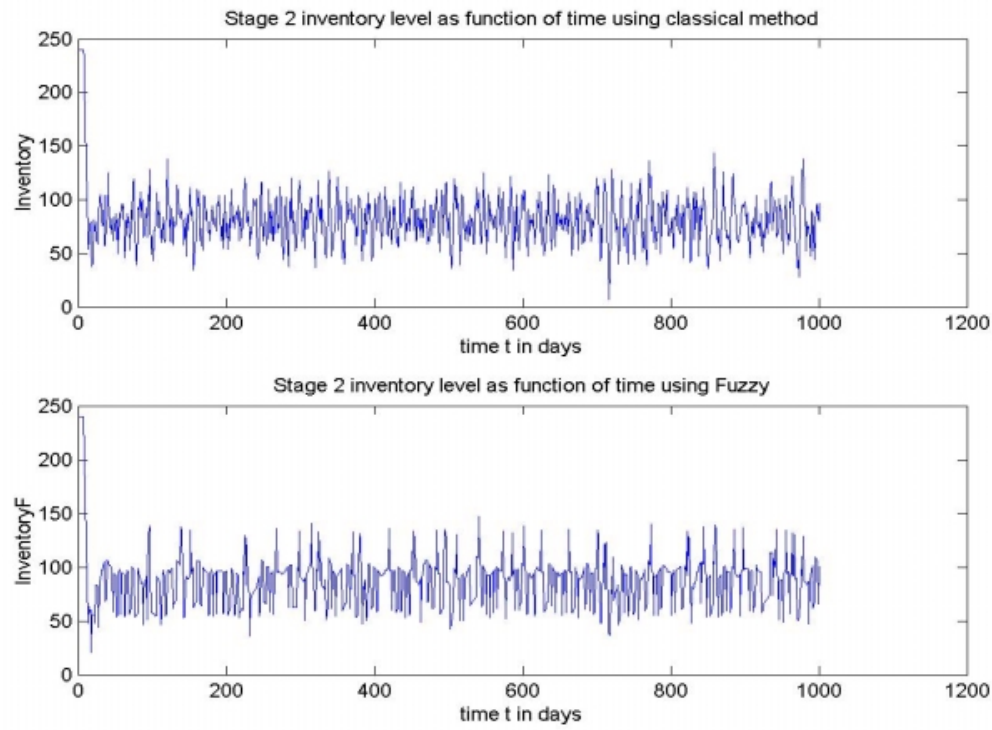

Fig. 6. Case 2: Inventory curve of stage 2 using fuzzy and classical approach 

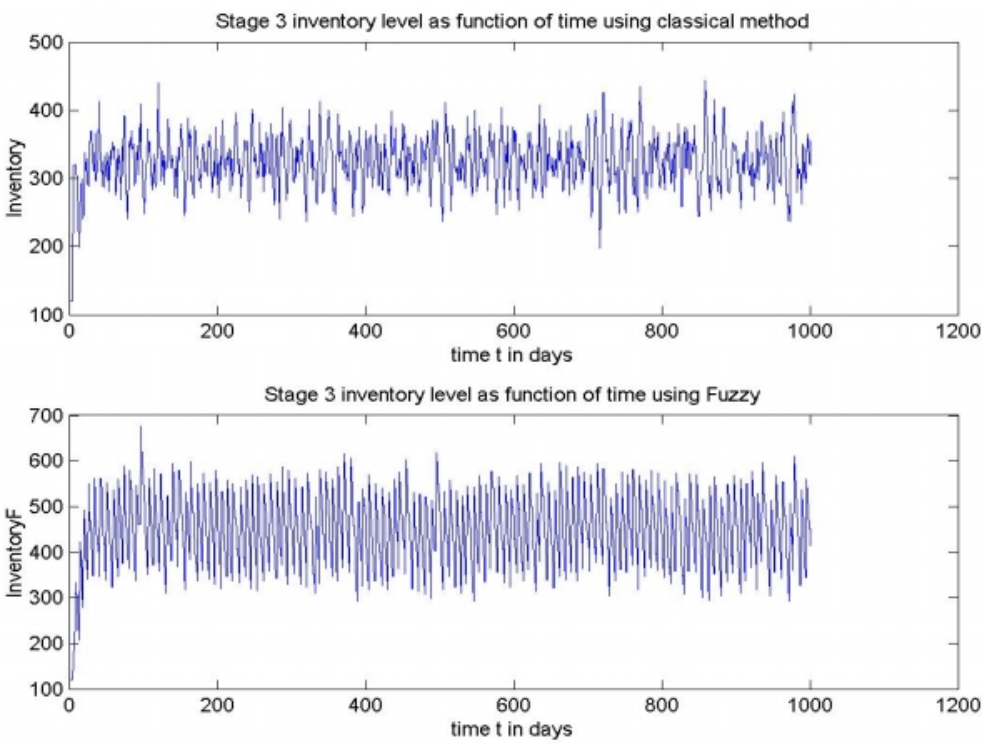

Fig. 7. Case 2: Inventory curve of stage 3 using fuzzy and classical approach
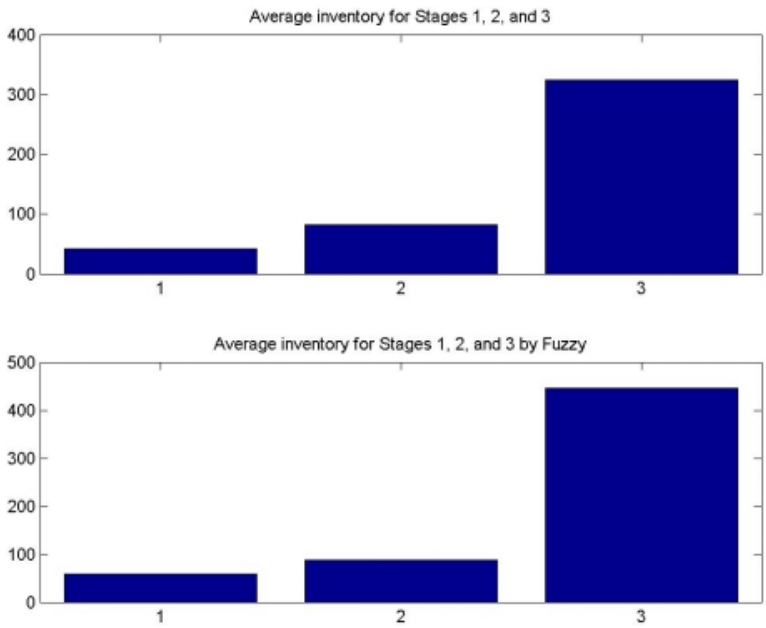

Fig. 8. Case 2: Average inventory using fuzzy and classical approach

To compare the fuzzy and classical inventory control systems based the above cases. This was done by comparing the total cost, excluding ordering cost, of each system using identical demand streams for some periods for two cases. Based on the uniform demand distribution, we ran simulation of 360 periods to evaluate the per- 
formance for the two cases. We started with the control rules as the Table 1 and fuzzy operator as above. See Figure 4 -8, Tables 2 and 3 for explicit results.

Table 2 and Table 3 compare the simulation between the fuzzy inventory system and the classical inventory system. Clearly, for the fuzzy inventory system, but its total cost is much lower because its number of times of order is much less than using the classical inventory system even though its average inventory is higher than the classical approach. Also its inventory level is much stable than using the classical inventory system. The simulation has demonstrated that the fuzzy logical approach can successfully tune a fuzzy controller using tuning membership function. The fuzzy controller can cope with the imprecise information, its matrix can be improved by the experts based on the experiences.

\section{Conclusion}

In this paper we have provided a simple fuzzy logic approach for inventory control for the supply chain environment subject to uniform distribution demand. Our approach explicitly accounts for the differences from the classical approach in design. Based on our simulation studies we see that the approach yields benefits for different levels of variability in the in a supply chain environment, and fuzzy logical shows a powerful tool for inventory control in supply chain. However, fuzzy rules must be chosen carefully. Improperly chosen rules and parameters can result in worse performance than the classical systems. The proper-tuned fuzzy control system is superior to its classical control system when intelligently implemented. Therefore, the fuzzy logic approach is fairly robust. This is a significant attribute of our approach as it increases the viability of its application to inventory problems.

\section{References}

1. Shervais, S. Shannon, T.T.: Improving theoretically-optimal and quasi-optimal inventory and transportation policies using adaptive critic based approximate dynamic programming. Neural Networks, 2001. Proceedings. IJCNN 01. International Joint Conference on, Volume: 15-19 July 2001, Washington, DC, USA. Page(s): 1008-1013 vol.2

2. J.B.G.FRENK.: 'INVENTORY CONTROL'. (1999),

3. Hamdy A.Taha.: 'Operations Research, An introduction', Macmillan Publishing Company, 15th Edition. New York

4. Barbara Ballard, Andrew Zozom Jr.: A Comparison of Inventory Systems: (Q, r) vs. Fuzzy Control. OR591: Fuzzy Optimization and Decision Making. (7, May.1996).

5. J E Beasley.: http://www.ms.ic.ac.uk/jeb/or/invent.htmlOR-Notes.

6. http://www.uh.edu/ djwells/guidelines/guidelines.html

7. Guruprasad Pundoor.: Supply Chain Simulation Models for Evaluating the Impact of Rescheuling Frequencies. MASTER'S THESIS, the University of Maryland, Sep, 2002. 